\title{
The effects of span-wise and chord-wise flexibility on the aerodynamic performance of micro flapping-wing
}

\author{
YANG WenQing ${ }^{*}$, SONG BiFeng, SONG WenPing \& WANG LiGuang \\ School of Aeronautics, Northwestern Polytechnical University, Xi'an 710072, China
}

Received November 24, 2011; accepted February 13, 2012

\begin{abstract}
The flexibility of flapping-wing strongly affects the aerodynamic performance of Flapping-wing Micro Air Vehicle (FMAV), and the deformations in span-wise and chord-wise directions are coupled together in flight. In this study, the flexible deformation is formulated in span-wise and chord-wise separately in order to analyze its effects on aerodynamic behavior. The preconditioned Navier-Stokes equations based on chimera grid are used in the computational fluid dynamics method to study the aerodynamic effects caused by flexible deformation, and the simulation results are compared with experimental test to illustrate the capability of above method. Based on our results, it is clearly showed that the span-wise flexible deformation should be limited in a small range to achieve higher aerodynamic performance and the chord-wise deformation could enhance the aerodynamic performance. The results also suggest that FMAV designers should design the flapping-wing with high stiffness leading edge to limit the span-wise deformation, and more flexible chord ribs to keep chord-wise deformation in suitable range.
\end{abstract}

FMAV, flexible flapping-wing, Navier-Stokes equations, chimera grid, span-wise deformation, chord-wise deformation

Citation: Yang W Q, Song B F, Song W P, et al. The effects of span-wise and chord-wise flexibility on the aerodynamic performance of micro flapping-wing. Chin Sci Bull, 2012, 57: 2887-2897, doi: 10.1007/s11434-012-5249-1

It is well known that the natural flyers are flying with flapping wings, such as flying birds, insects and bats. The Flapping-wing Micro Air Vehicle (FMAV) is a kind of new pattern bionic air vehicle which can improve the low Reynolds number aerodynamic performance under low flight speed and small vehicle size conditions. For this advantage, FMAV has gained wide attraction of researchers in different countries. In what follows we provide some representative productions: Figure 1(a) shows the "Hummingbird" of America (http://www.chinanews.com/gj/2011/02-18/2852561. shtml), which was reported on February 18, 2011; Figure 1(b) shows the "Smart bird" of Germany (http://www.china. com.cn/v/news/2011-04/04/content_22290407.htm), which was reported on April 4, 2011; and Figure 1(c) shows the "Dove" of China (http://www.avicone.com/Article_Show. asp?ArticleID=16902), which was reported on September 24, 2011.

*Corresponding author (email: yangwenqing @ nwpu.edu.cn)
These FMAVs shows the development of aerodynamics and Micro-Electro-Mechanical Systems (MEMS) technology. However, their flight capabilities are still weak compared with the natural creatures. To improve the flight capability of FMAV, the corresponding aerodynamic mechanism still needs to be further investigated.

Many researchers keep concentrating in this field in the recent decade. A lot of prototypes employed the flapping flight have been developed by many researchers. To find out the inbeing of flapping flight aerodynamics, they did a lot of investigations based on theoretical, experimental and numerical methods. Lin and $\mathrm{Hu}$ [1], Zhang et al. [2], Sun and Tang [3], Xie et al. [4], and Yang et al. [5], have determined the basic performance of flapping-wing by CFD. Shao et al. [6] and Jones et al. [7-9], have performed some basic behaviors of flapping-wing by using the wind tunnel experiments. The above research obtained some basic rules of flapping-wing flight.

The flexible wing is applied widely by MAV designers 
(a)

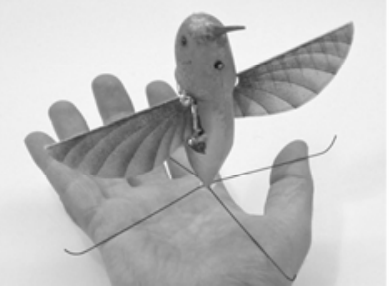

(b)

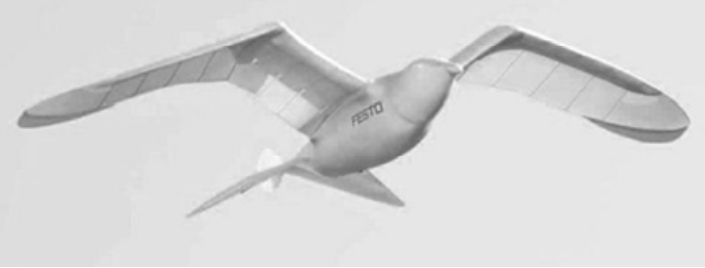

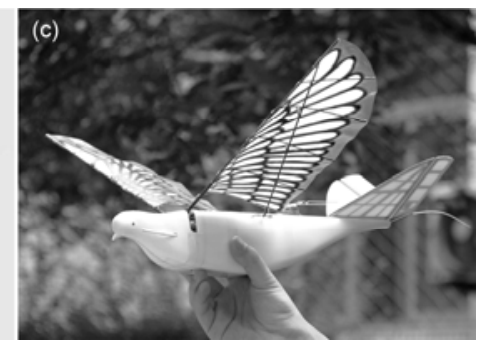

Figure 1 Representative FMAVs. (a) "Hummingbird" of America; (b) "Smart bird" of Germany; (c) "Dove" of Northwestern Polytechnical University of China.

for its good stability and anti-gust ability, which is used both in the flapping-wing and fixed-wing MAVs. The wings of three typical FMAVs mentioned in Figure 1 are also flexible. However, the flexible flapping-wing has very complex characteristics because of the loop coupling influence among the structure deformation, aerodynamic force, and inertial force. Research shows that the bird can accommodate itself as airflow changes by deforming the wings actively to take advantages of aerodynamic performance, but the wing structure of FMAV always deforms passively, which is different from bird.

The structures of flapping wing are always light and elastic, and driven by flapping machine in the wing root, which deforms under the pressure of aerodynamic force and inertial force. Although the wing of some FMAVs can deform actively by control instruments, the mass consumption may not affordable; therefore, the active deformation is rarely used in the FMAV.

Wu et al. [10] studied the passive structural deformation of flexible membrane flapping-wing, and summarized some principles of passive deformation effects on the aerodynamic performance of FMAV. They revealed that the wing could produce thrust effectively only under some certain modes of passive aero-elastic deformation. Chimakurthi et al. [11] proposed a computational aero-elasticity framework to analyze FMAV, and they thought the span-wise flexibility had a favorable impact on the thrust generation. Ho et al. [12] concluded the relationship between flapping wing structure and aerodynamic behavior, and they stated that the lift relied heavily on wing flexibility.

These investigators have revealed some effects of flexible structural deformation on the aerodynamic behavior. However, the span-wise and chord-wise deformations in these studies were coupled together, and the separate effects of span-wise or chord-wise deformation were not considered, which would cause some difficulties when the designer wanted to know the structural stiffness distributing at the beginning of FMAV design process. The problem would be explored in this paper.

This work is organized as follows. At first, the flapping motion is described in four modes: (1) pure rigid flapping without any deformation, (2) separate span-wise deformation flapping, (3) separate chord-wise deformation flap- ping, and (4) combining span-wise and chord-wise deformation flapping. Then, the above deforming motions are investigated using the CFD method which is validated by comparing with the experimental results. Based on the numerical results, the span-wise and chord-wise deformation effects are further analyzed. Finally, results and conclusions are given and discussed.

\section{Flapping-wing motion}

The flapping-wing motion is defined in this section. Firstly, the single-degree of freedom (DOF) motion of pure rigid flapping motion is described as basic state

$$
\psi(t)=\psi_{m} \cos (\omega t) .
$$

The reduced frequency is

$$
k=\frac{\omega c}{2 U_{\infty}}
$$

where $\psi$ is the flapping angle in the $t$ time step, $\psi_{m}$ is the maximum flapping angle, $\omega$ is the frequency, $c$ is the chord length, and $U_{\infty}$ is the freestream velocity. The pure rigid flapping motion is shown in Figure 2(a).

Next, the span-wise and chord-wise deformations will be added into the basic single-DOF flapping motion.

Wing deforming laws are based on the rational assumption: the deformation is proportional to the flapping velocity. The wing deformation always happen passively, and main causes induced the structure deformation are the aerodynamic force and the inertial force. When the flapping frequency is low as bird's flight, the inertial force of wing could be ignored because it is far less than the aerodynamic force. Thus, the aerodynamic force is the main reason caused the wing deformation. Therefore, it is rational that the aerodynamic force is thought to be approximately proportional to the flapping velocity in basic theoretical research, which will be explained in Section 3.

Let $\phi_{s}$ and $\phi_{c}$ denote the span-wise and chord-wise deformation angles respectively. The flapping motion with span-wise deformation can be expressed as

$$
\psi(z, t)=\psi_{m} \cos (\omega t)+\phi_{s}(z) \sin (\omega t),
$$




$$
\phi_{s}(z)=\phi_{s, m} \times \frac{z}{b}
$$

where $\phi_{s}$ is the span-wise deformation angle in the $z$ position, $\phi_{s, m}$ is the maximum span-wise deformation angle, and $b$ is the half span length. The span-wise deformation process is shown in Figure 2(b). It looks like wingspan camber when the span-wise deformation occurs.

Similarly, the chord-wise deformation can be expressed as

$$
\begin{gathered}
\alpha(z, t)=\alpha_{0}-\phi_{c}(z) \sin (\omega t), \\
\phi_{c}(z)=\phi_{c, m} \times \frac{z}{b},
\end{gathered}
$$

where $\alpha$ is the instantaneous angle of attack of $z$ position in the $t$ time step, $\alpha_{0}$ is the mean angle of attack, $\phi_{c}$ is the chord-wise deformation angle in the $z$ position, and $\phi_{c, m}$ is the maximum chord-wise deformation angle. The chord- wise deformation process is shown in Figure 2(c). When the chord-wise deformation occurred, the airfoil is thought to be rigid. Therefore, the wing with chord-wise deformation is shown as wing twisting.

The deformations of span-wise and chord-wise are independent to each other in kinematics. In the combining research, the deformation laws are the same as separate cases, but deformations happen simultaneously. The combining deformation process is shown in Figure 2(d).

\section{Numerical method}

The preconditioned RANS (Reynold-Averaged NavierStokes equations) [13] can be written as

$$
\boldsymbol{\Gamma}^{-1} \frac{\partial \boldsymbol{W}_{\boldsymbol{T}}}{\partial \tau}+\frac{\partial \boldsymbol{W}}{\partial t}+\frac{\partial\left(\boldsymbol{E}-\boldsymbol{E}_{v}\right)}{\partial x}+\frac{\partial\left(\boldsymbol{F}-\boldsymbol{F}_{v}\right)}{\partial y}+\frac{\partial\left(\boldsymbol{G}-\boldsymbol{G}_{v}\right)}{\partial z}=0
$$
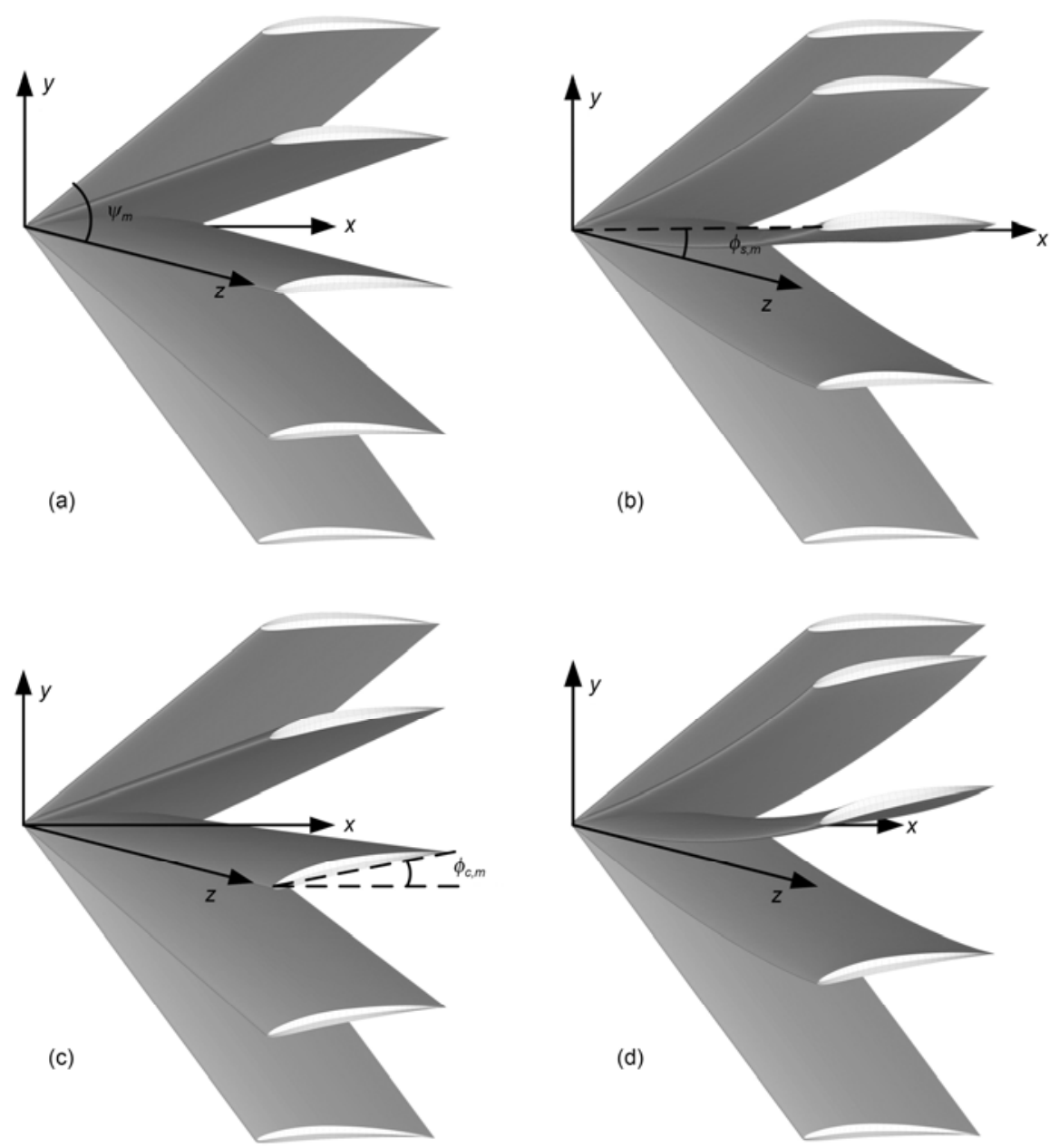

Figure 2 Schematics of flapping motion. (a) Pure rigid flapping motion in single-DOF of flapping-wing (motions are symmetric in down-stroke and up-stroke); (b) span-wise deformation process of flapping-wing in down-stroke (motions are antisymmetric in down-stroke and up-stroke); (c) chord-wise deformation process of flapping-wing in down-stroke (motions are antisymmetric in down-stroke and up-stroke); (d) combining span-wise and chord-wise deformation process of flapping-wing in down-stroke (motions are antisymmetric in down-stroke and up-stroke). 
where $\Gamma^{-1}$ is preconditioning matrix, $\tau$ is pseudo time, $t$ is true time, $\boldsymbol{E}, \boldsymbol{F}, \boldsymbol{G}$ are inviscous flux, $\boldsymbol{E}_{v}, \boldsymbol{F}_{v}, \boldsymbol{G}_{v}$ are viscous flux.

The spatial discretization is characterized by a secondorder cell-center method for finite volumes. And a fivestage Runge-Kutta scheme is employed to achieve convergence of the solution by integration with respect to time. For the unsteady flows an implicit dual time-stepping scheme is used. The implicit residual smoothing method is employed to accelerate the convergence. For calculating the turbulence flows, the Spalart-Allmaras one-equation turbulent model is applied.

Figure 3 shows the grid system of flapping-wing based on the chimera grid method. Figure 4 shows the Flapping-wing surface grid. There are two level grids: stationary background grid and moving flapping-wing grid. During the calculating process, the wing grid is regenerated in every time step. Figure 3(a) shows the flapping wing in neutral position while Figure 3(b) shows the flapping wing in the top position.

The corresponding relationship between background grid and wing grid needs to be confirmed in every time step that is the pre-processing. In this study, the distance-decreasing searching (DDS) method is used to deal with the pre-processing, which is based on a simple theory that the corre-
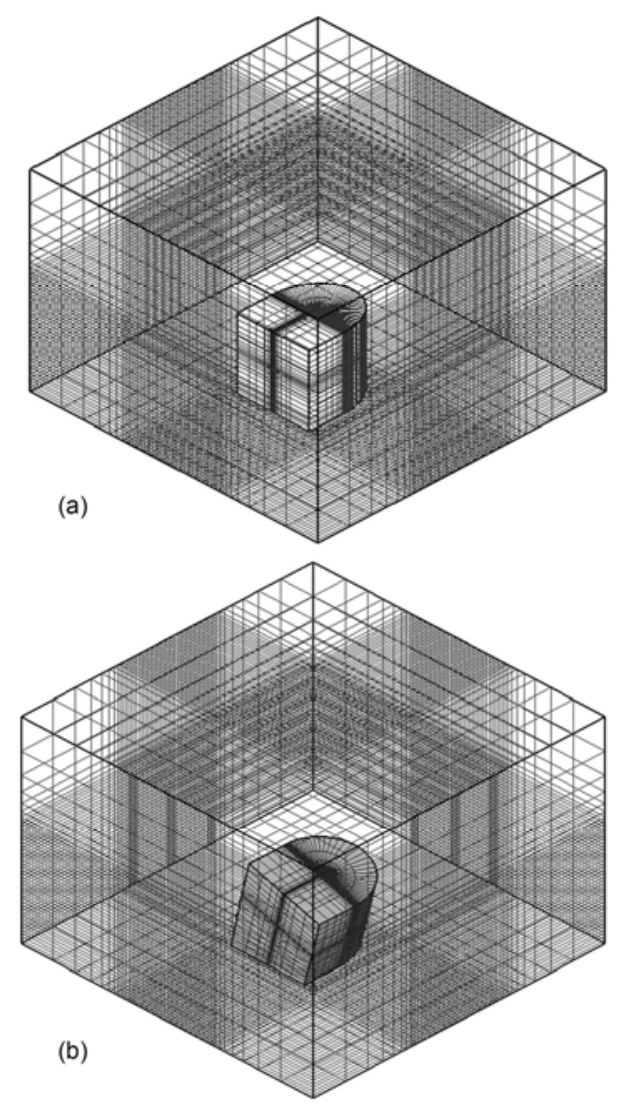

Figure 3 Chimera grid system of flapping-wing. (a) The flapping wing in the neutral position; (b) the flapping wing in the top position.

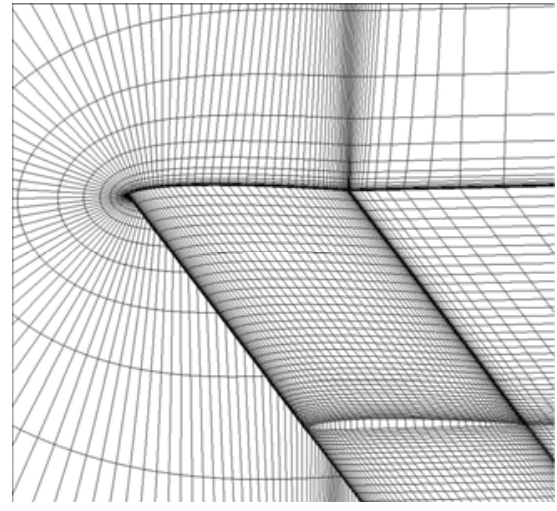

Figure 4 Flapping-wing surface grid.

sponding cells of any two overlapped grids are in nearest distance.

The algorithm of DDS method is as following.

Step 1 Given a point $A$ in the target grid, and a point $B$ from the source grid, calculate the distance between point $A$ and point $B$, denoted by $l_{A B}$.

Step 2 Calculate the distance between point $A$ and those points around point $B$, choose the nearest point denoted by $C$, the distance between point $A$ and point $C$ is denoted by $l_{A C}$.

Step 3 Compare the distances $l_{A B}$ and $l_{A C}$ : if $l_{A B}$ is not greater than $l_{A C}$, the point $B$ is the point nearest to point $A$ in source grid region $\Omega$, stop searching; if $l_{A B}$ is greater than $l_{A C}$, let $C$ replace of point $B$, turn to step 2 and continue.

This process is the algorithm of DDS method, the name got because the distance between two points is decreasing till the closest point is found. The method is efficient, reliable and simple in hole-cutting and interpolating donor cells searching. Figure 5 is the sketch of this method, which shows an overlapping field of Cartesian grid and curvilinear grid.

The cell center value is needed when using the cell-center scheme finite volume method. Here, let $P$ denotes an interpolated cell in curvilinear grid. Then, the interpolating donor

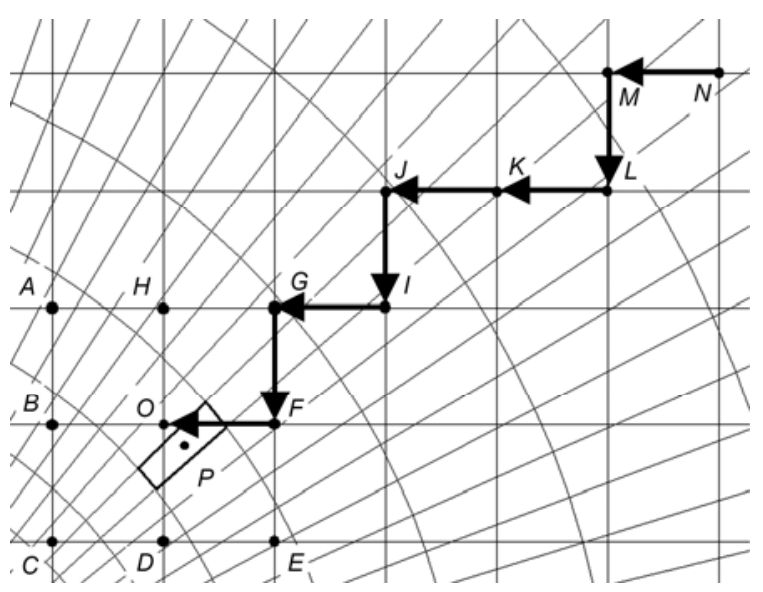

Figure 5 Sketch of DDS method. 
cells in Cartesian grid should be found out, which are the cells $A B O H, B C D O, O D E F$ and $H O F G$. If the point $O$ is found, the four donor cells will be fixed. We can begin from any point, taking the point $N$ for example, the path from $N$ to $O$ is $N \rightarrow M \rightarrow L \rightarrow K \rightarrow J \rightarrow I \rightarrow G \rightarrow F \rightarrow O$ searched by the DDS method.

In traditional way, the quantity of searching points should be $O(i \times j)$ in 2D grids, and $O(i \times j \times k)$ in 3D grids (here $i, j$, $k$ are cell numbers of curvilinear gird). From the sketch of Figure 5, we know the search path of DDS method is on a curve, similar to linear search. The number of search points is less than 4 times the number of diagonal cells in 2D grids, or 6 times the number of diagonal cells in 3D grids. That is, the quantity of searching points of the DDS method is $O\left(4 \sqrt{i^{2}+j^{2}}\right)$ in 2D grids, and $O\left(6 \sqrt{i^{2}+j^{2}+k^{2}}\right)$ in $3 \mathrm{D}$ grids. Therefore, this method can be thought to be quasi-linear. As the cell number is huge, the DDS method can work with high efficiency.

The original hole-boundary and the artificial hole-boundary in pre-processing of chimera grid system are shown in Figure 6.

\section{Validation of numerical method}

The experimental state in [14] is close to the bird-like scope of this study, so it is used to validate the numerical method.

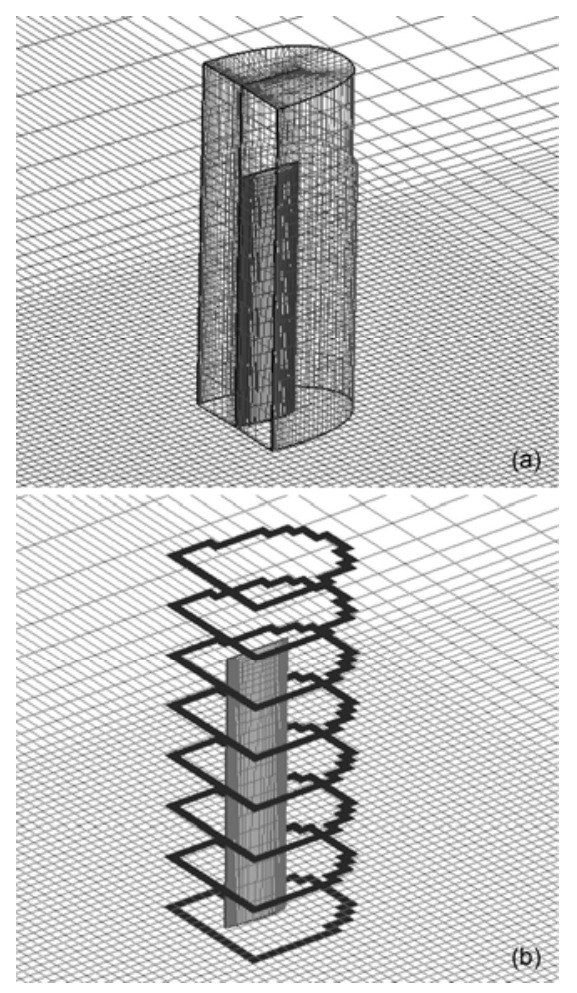

Figure 6 Schematic of the hole-cutting in pre-processing of chimera grid system. (a) Original hole-boundary; (b) artificial hole-boundary.
The flapping-wing is rectangle surface with airfoil NACA8318. Flapping motion accords to the law described in Figure 2(a) and eq. (1). And other parameters are: chord length is $76 \mathrm{~mm}$; span length of the flapping-wing is $305 \mathrm{~mm}$; wind velocity is $21.4 \mathrm{~m} / \mathrm{s}$; Reynolds number is $10.7 \times 10^{4}$; angle of attack is $0^{\circ}$; plunging angle is $45^{\circ}$; flapping frequency is $3.3 \mathrm{~Hz}$.

Flapping-wings are the motor of FMAV, which generate both lift and thrust simultaneously - the lift makes vehicle stay in the air and the thrust makes vehicle fly forward. For this reason, this study focus on the lift and thrust characteristics, and the lift coefficient $\mathrm{Cl}$ and the thrust coefficient $\mathrm{Ct}$ will be studied in flapping cycle and in averaged value.

Figure 7 shows the variation of lift and thrust coefficient in a flapping cycle: the solid line are got by RANS equations and the dotted line are from experiments of [14]. Known from Figure 7, the computational lift coefficients are in good accordance with the experiment, but the computational thrust coefficients are not very close to the experiment.
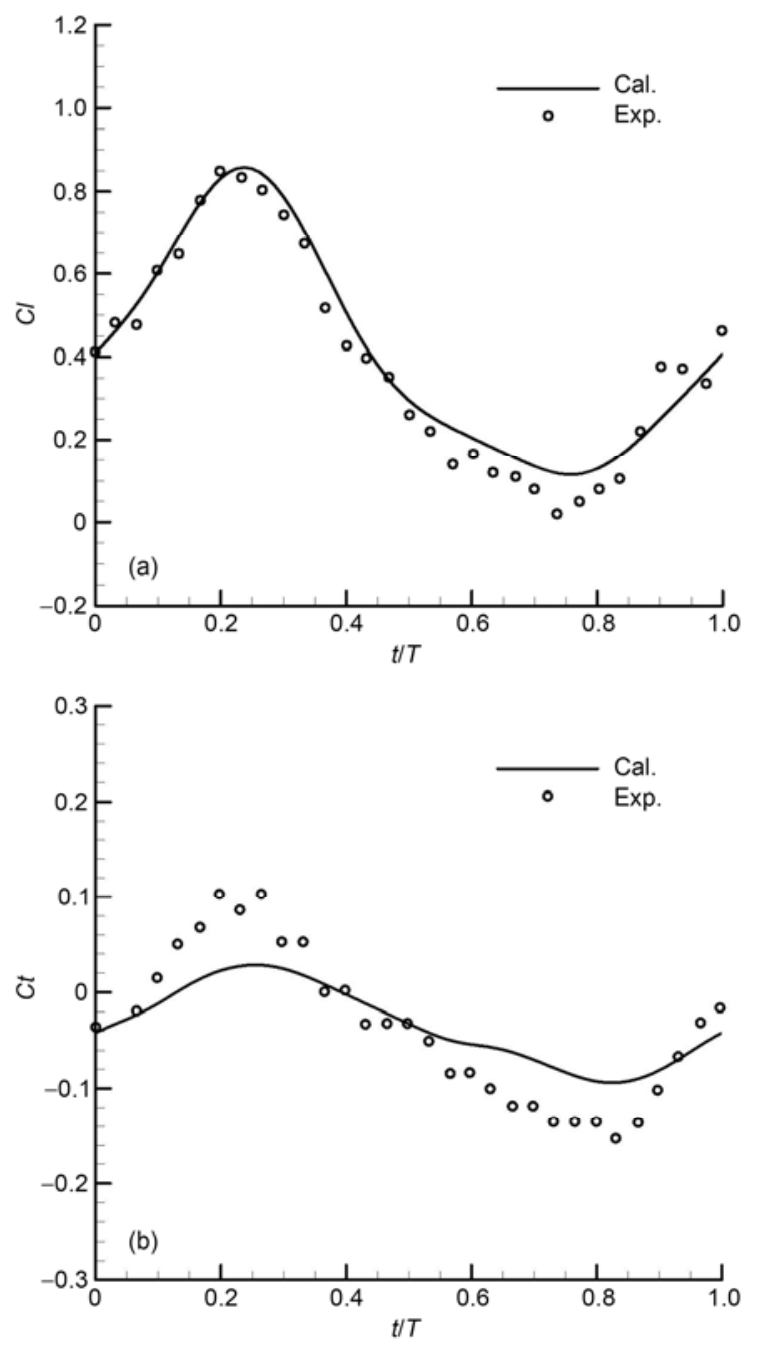

Figure 7 Results comparison between calculated results and experiment results in reference. (a) Lift coefficients $C l$ vs. $t / T$ in a flapping cycle; (b) thrust coefficients $C t$ vs. $t / T$ in a flapping cycle. 
Although there are deviations, the computational thrust coefficients and experiment are still have similar trend and averaged value. Considering about the difficulties in thrust experiment and calculation, it is rational that the computational results are thought to be reasonable.

Figure 8 shows the relationship among wing flapping motion, flapping velocity and lift coefficient. The trend of flapping velocity is consistent to the lift coefficient, and the peak positions of lift coefficient curve are close to the peak positions of flapping velocity curve. Because the lift force is the main reason caused the structure deformation, the assumption of Section 1 could be reasonable that the structure deformation is proportional to flapping velocity.

\section{Results and discussion}

In this section, the effects of span-wise deformation and chord-wise deformation are investigated. The flapping-wing used in calculation has a rectangle surface with airfoil of NACA4408, and other states parameters are: half aspect ratio is 4; Mach number is 0.03 ; Reynolds number is $10 \times 10^{4}$; flapping angle is $20^{\circ}$; angle of attack is $5^{\circ}$; reduced frequency is 0.4 . These flight parameters described above are close to medium birds, such as pigeon and pied magpie. Considering the airfoils of medium bird wings are all camber, and then the $4 \%$ camber airfoil is chosen in numerical
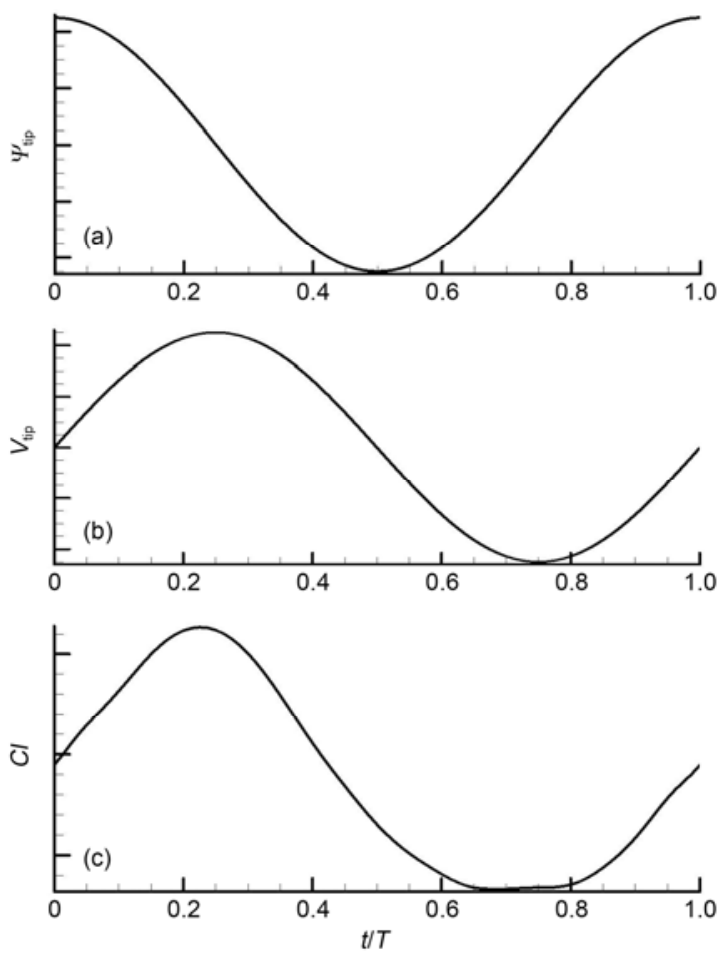

Figure 8 Relationship among wing flapping motion, flapping velocity and lift coefficient. (a) Wing tip position vs. $t / T$ in a flapping cycle; (b) wing tip velocity vs. $t / T$ in a flapping cycle; (c) lift coefficient vs. $t / T$ in a flapping cycle. simulation. The flexible deformation angle is supposed to vary from $0^{\circ}$ to $10^{\circ}$ thinking about the true deformation range and proportion.

In subsection 4.1, the effects of span-wise deformation are discussed in two ways. Firstly, an ideal model without chord-wise deformation is calculated. Secondly, the spanwise effects in the condition with certain chord-wise deformation are discussed. The former ideal model shows the pure span-wise deformation effects, and the latter results show the impact of variable span-wise flexibility effects when chord-wise flexibility is constant. Main arrangement of subsection 4.2 is similar to subsection 4.1, and the effects of chord-wise deformation are researched. Combining effects of span-wise and chord-wise deformation are discussed in subsection 4.3, which are based on the results of former two subsections. And then, subsection 4.4 shows the flowfields pressure variation of a flapping cycle. The span-wise and chord-wise deformation effects are explained in a visual way.

\subsection{Span-wise deformation effects}

Figure 9 shows the span-wise deformation effects on the aerodynamic performance when the chord-wise deformation angle is $0^{\circ}$. This is an ideal state without chord deformation.

As the span-wise deformation angle increases, Figure 9(a) shows that the fluctuating range of lift coefficient curve will also increase and the phase will delay a little; Figure 9(b) shows that the phase of thrust coefficient curve will delay and the shape will change; Figure 9(c) shows that the average lift will increase a little and then decrease a lot; Figure 9(d) shows the similar trend to the average lift.

Next, the span-wise deformation effects are studied in the situation of constant chord-wise deformation angle. Figures 10 and 11 show the effects when the chord-wise deformation angles are $5^{\circ}$ and $10^{\circ}$, respectively.

The effect in Figure 10 is similar to Figure 9. However, Figure 11 shows different effects, where the averaged lift and thrust coefficients will increase with the increase of the span-wise deformation angle.

Figures 9-11 show that the span-wise deformation angle effects on the aerodynamic performance are complex. When the chord-wise deformation angle is in small range, the larger span-wise deformation can leads to the worse lift and thrust characteristics as shown in Figures 9 and 10. When the chord-wise deformation angle is in larger range, the larger span-wise deformation can improve the lift and thrust characteristics slightly as shown in Figure 11.

Figures 9-11 indicate that (1) when the chord-wise deformation is large, the span-wise deformation may also be larger; and (2) the span-wise deformation should be less than the chord-wise deformation.

\subsection{Chord-wise deformation effects}

The chord-wise deformation effects on the aerodynamic 

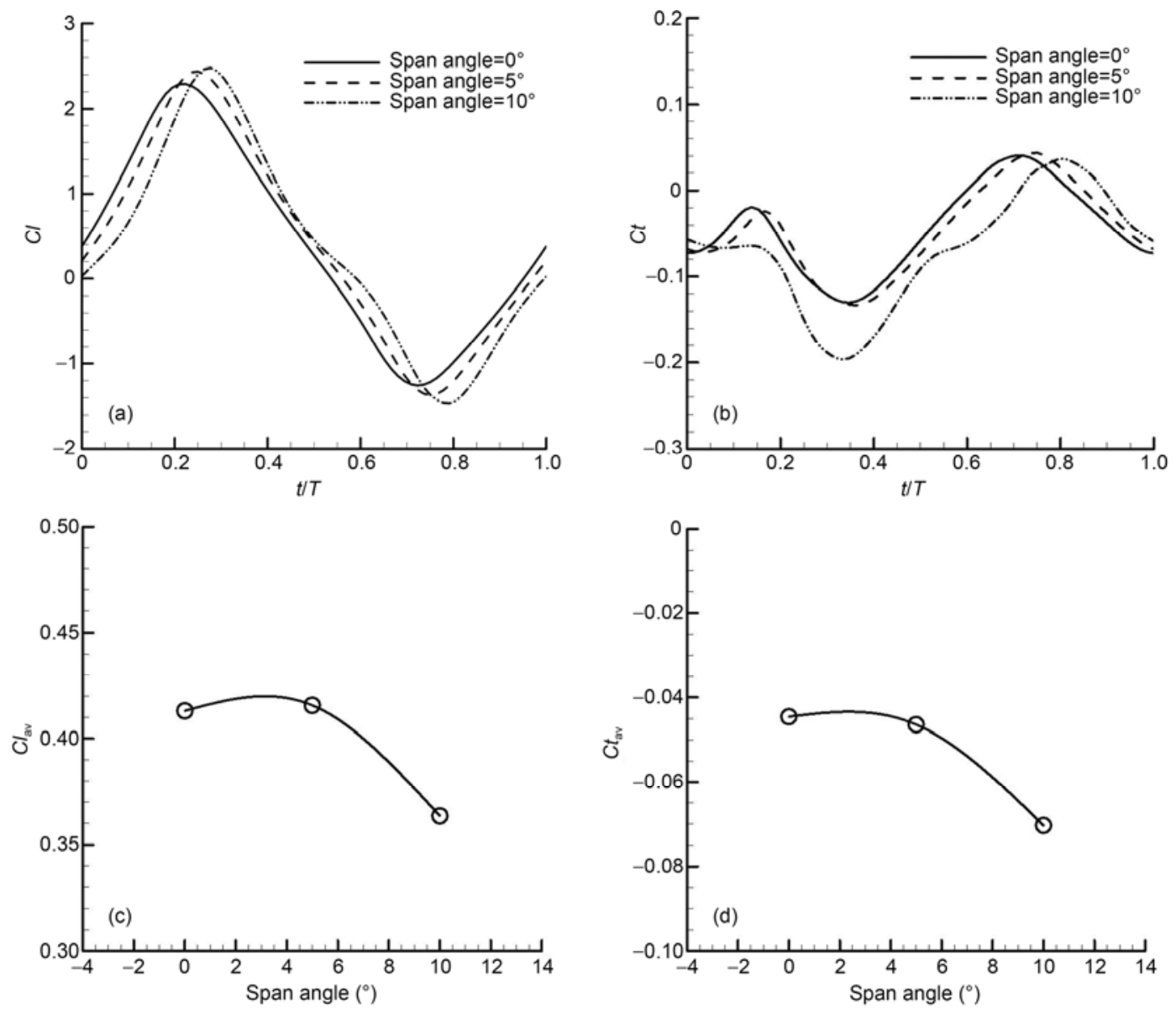

Figure 9 The span-wise deformation effects when the chord-wise deformation angle is $0^{\circ}$. (a) Lift coefficient $C l$ vs. $t / T$ in a flapping cycle; (b) thrust coefficient $C t$ vs. $t / T$ in a flapping cycle; (c) average lift coefficient vs. span-wise deformation angle; (d) average thrust coefficient vs. span-wise deformation angle.
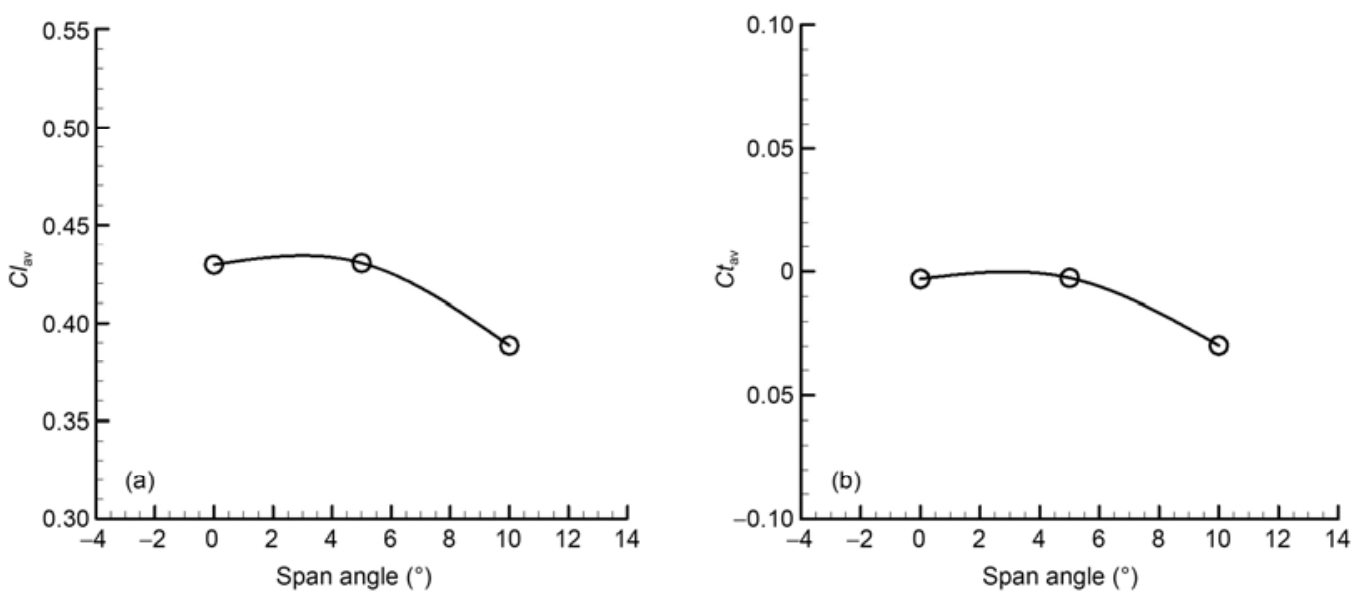

Figure 10 The span-wise deformation effects when the chord-wise deformation angle is $5^{\circ}$. (a) Average lift coefficient vs. span-wise deformation angle; (b) average thrust coefficient vs. span-wise deformation angle.

performance are shown in Figures 12-14 when the spanwise deformation angles are $0^{\circ}, 5^{\circ}$ and $10^{\circ}$, respectively.

The results of Figures 12-14 show that the large chordwise deformation angle has good effects: (1) the increasing of chord-wise deformation angle can improve the lift and thrust characteristics; (2) when the span-wise deformation is larger, the chord-wise deformation effects are stronger. These advantages of chord-wise deformation are because the direction of flow will deflect to backward under the deformation way described in this study. 

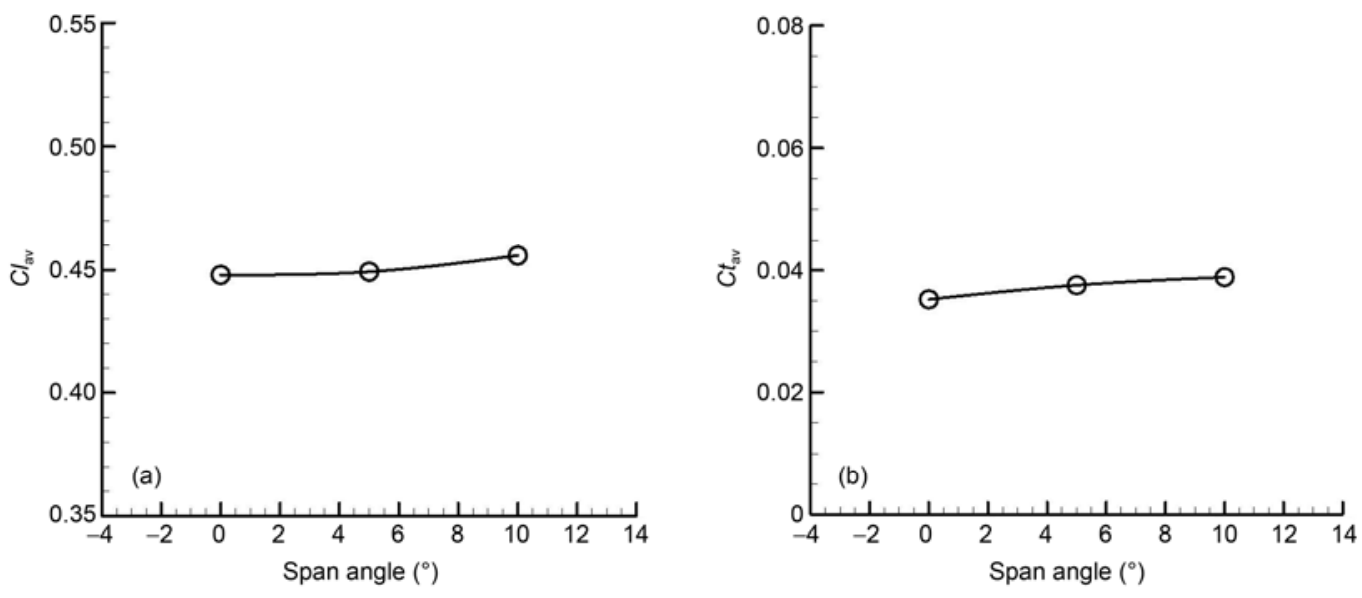

Figure 11 The span-wise deformation effects when the chord-wise deformation angle is $10^{\circ}$. (a) Average lift coefficient vs. span-wise deformation angle; (b) average thrust coefficient vs. span-wise deformation angle.
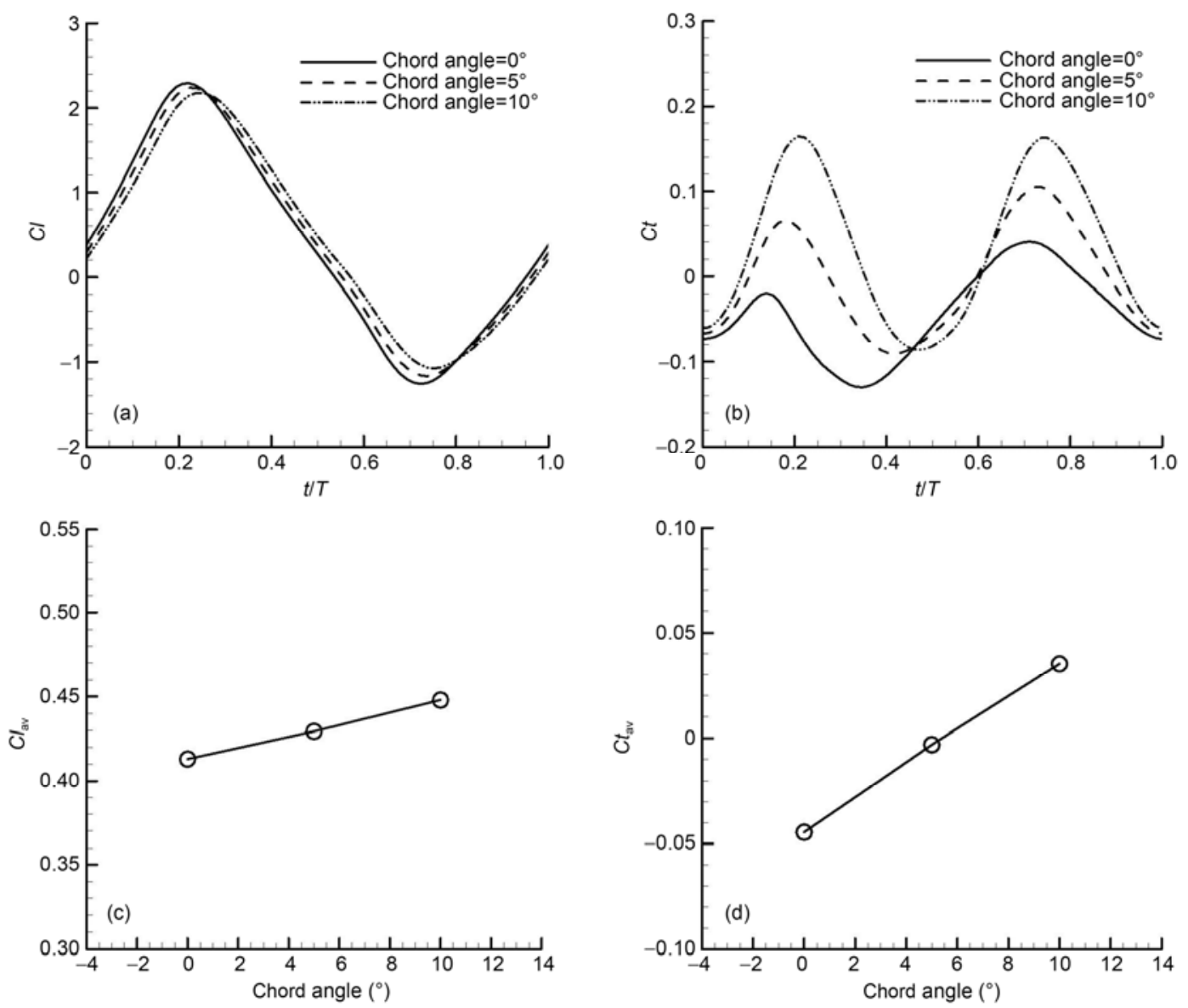

Figure 12 The chord-wise deformation effects when the span-wise deformation angle is $0^{\circ}$. (a) Lift coefficient $C l$ vs. $t / T$ in a flapping cycle; (b) thrust coefficient $C t$ vs. $t / T$ in a flapping cycle; (c) average lift coefficient vs. chord-wise deformation angle; (d) average thrust coefficient vs. chord-wise deformation angle.

\subsection{Combining analysis of span-wise and chord-wise deformation effects}

According to above analysis, the span-wise deformation should be small, while the chord-wise deformation should be large. Figures 9 and 10 tell us that the span-wise deformation should be less than $3^{\circ}$ when the chord-wise deformation is in small range. Figure 11 tells us that a little larger span-wise deformation is helpful when the chord-wise deformation is larger. Different from span-wise deformation, 

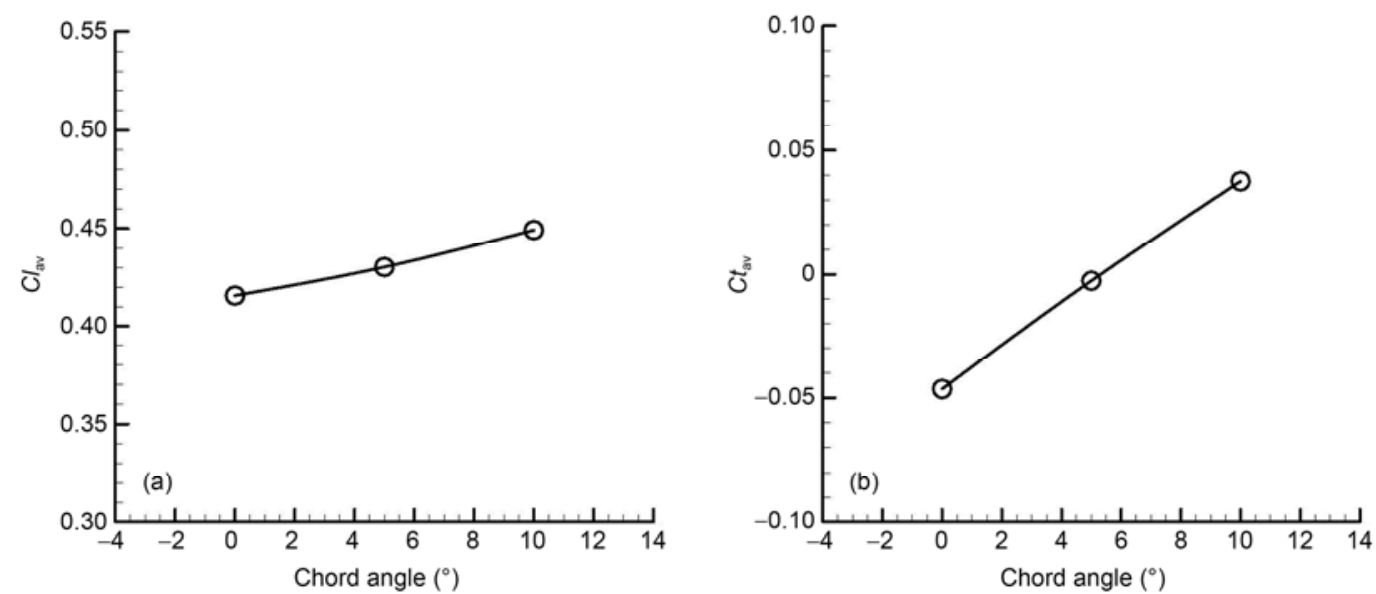

Figure 13 The chord-wise deformation effects when the span-wise deformation angle is $5^{\circ}$. (a) Average lift coefficient vs. chord-wise deformation angle; (b) average thrust coefficient vs. chord-wise deformation angle.
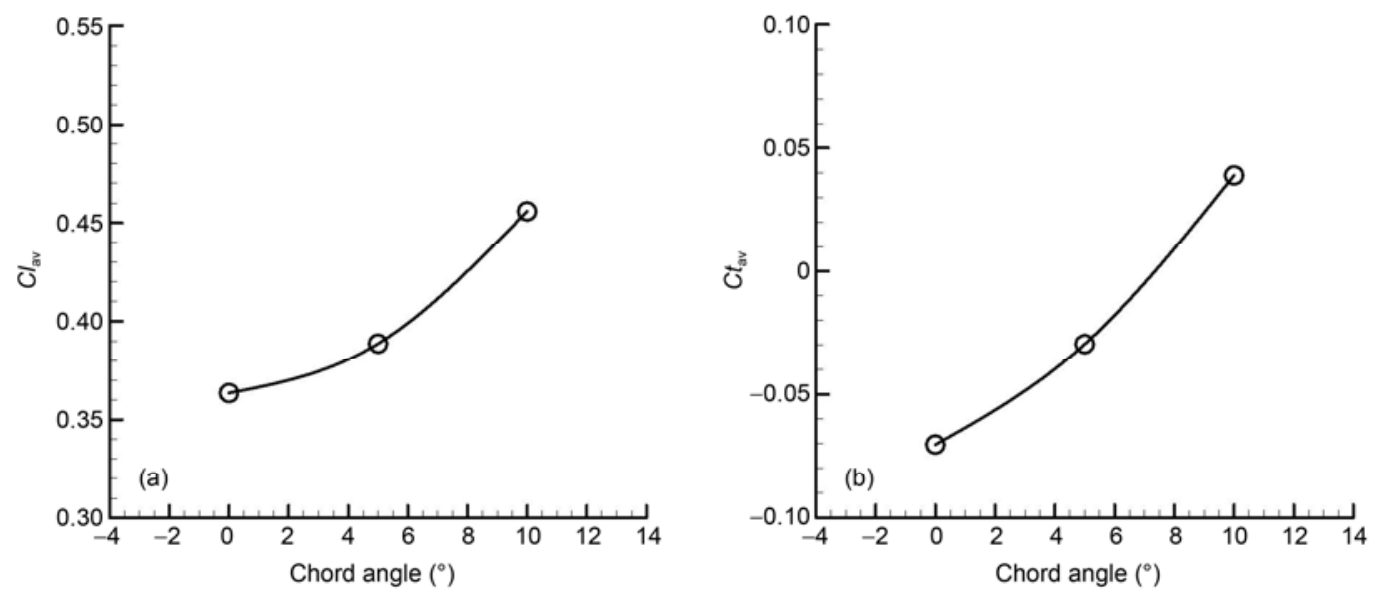

Figure 14 The chord-wise deformation effects when the span-wise deformation angle is $10^{\circ}$. (a) Average lift coefficient vs. chord-wise deformation angle; (b) average thrust coefficient vs. chord-wise deformation angle.

the chord-wise deformation has simple effects. Larger chordwise deformation can result in good aerodynamic performance when the chord-wise deformation is less than $10^{\circ}$.

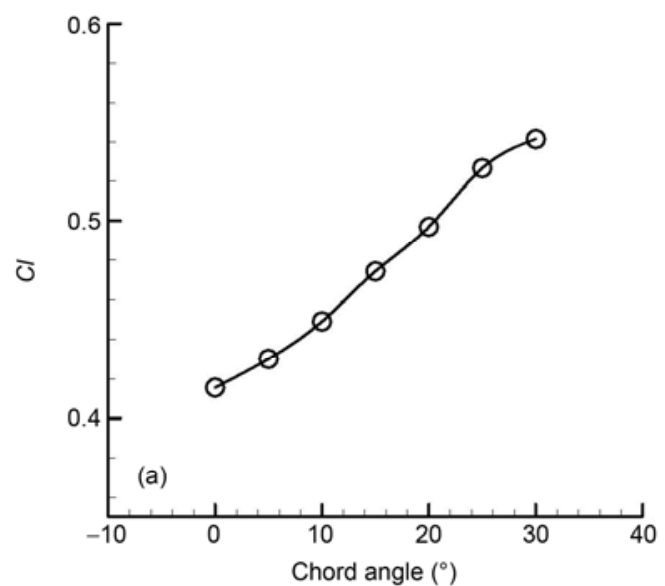

Figure 15 shows the effect of chord-wise deformation in a more large range when span-wise deformation angle is $5^{\circ}$. It is shown that the chord deformation should increase to

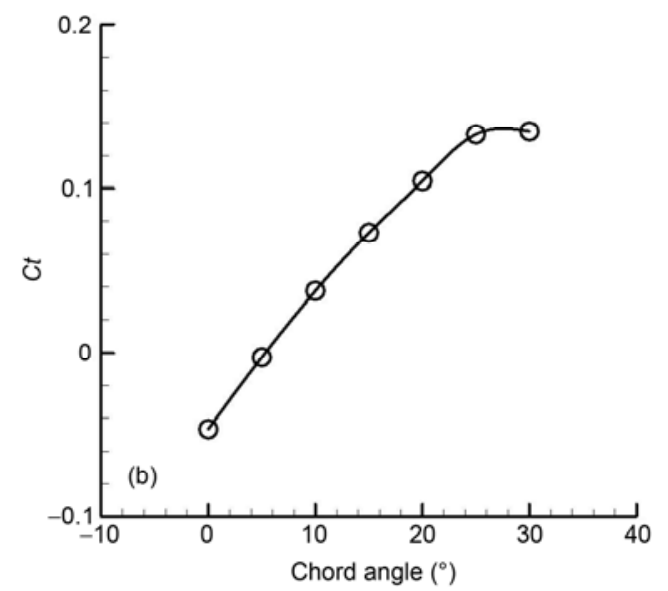

Figure 15 The chord-wise deformation effects when the span-wise deformation angle is $5^{\circ}$. (a) Average lift coefficient vs. chord-wise deformation angle; (b) average thrust coefficient vs. chord-wise deformation angle. 
$25^{\circ}$ under the condition of $5^{\circ}$ span-wise deformation angle. Therefore, the chord deformation angle can enhance aerodynamic performance in a certain range.

\subsection{Flowfield pressure analysis}

In this subsection, the flowfields pressure are analyzed, and the slice in the position of $75 \%$ span length is chosen to reflect the flowfields variation in a flapping cycle as shown in Tables 1 and 2. There are four situations: (1) pure rigid flapping motion, (2) flapping with rigid span and flexible chord-wise deformation, (3) flapping with rigid chord and flexible span-wise deformation, and (4) flapping with both span-wise and chord-wise flexible deformation. Table 1 shows the flowfields pressure variation of down-stroke process and Table 2 shows the up-stroke process. The first rows

Table 1 Flowfields pressure in the down-stroke process at the slice of $75 \%$ span length

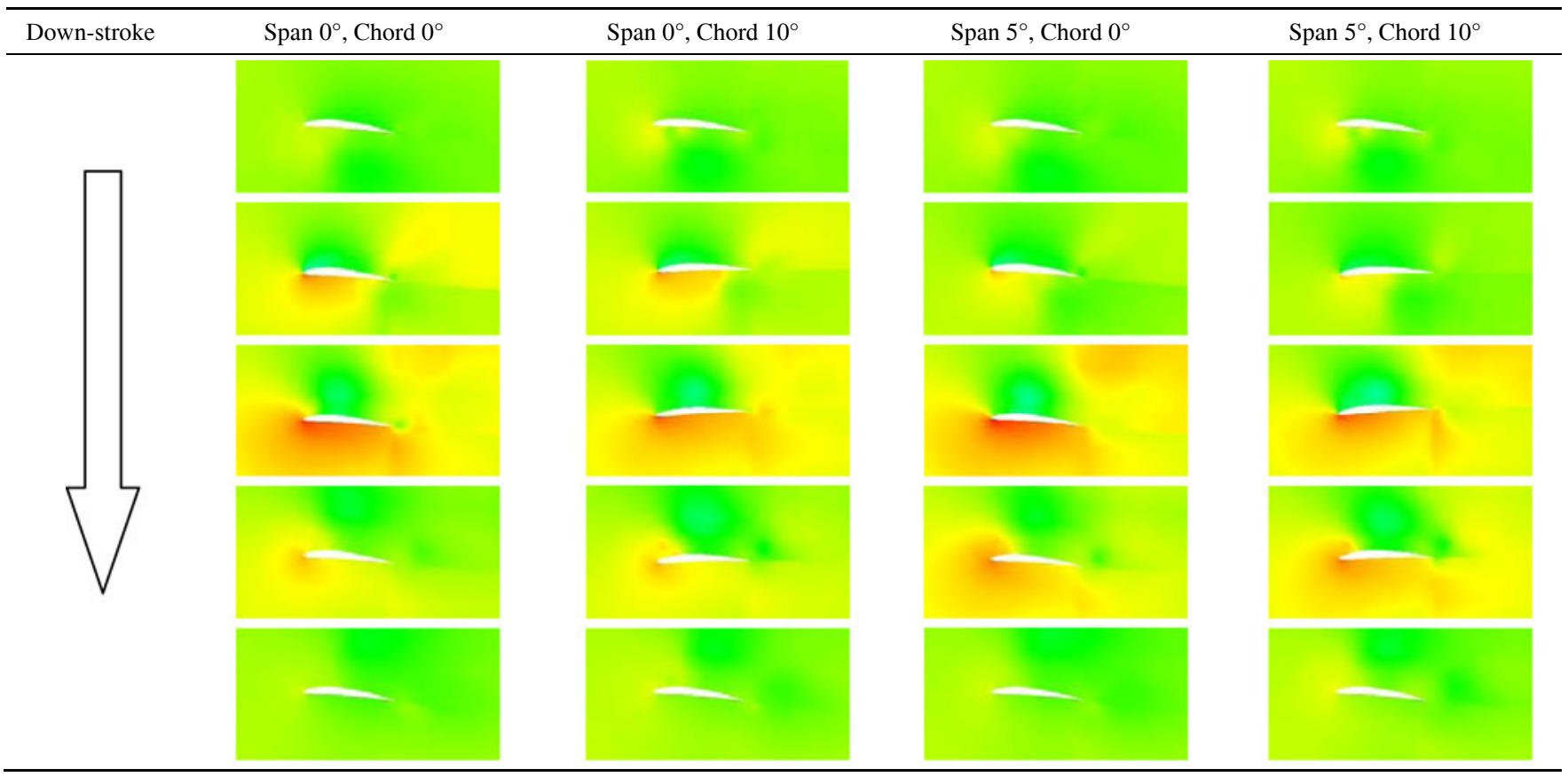

Table 2 Flowfields pressure in the up-stroke process at the slice of $75 \%$ span length

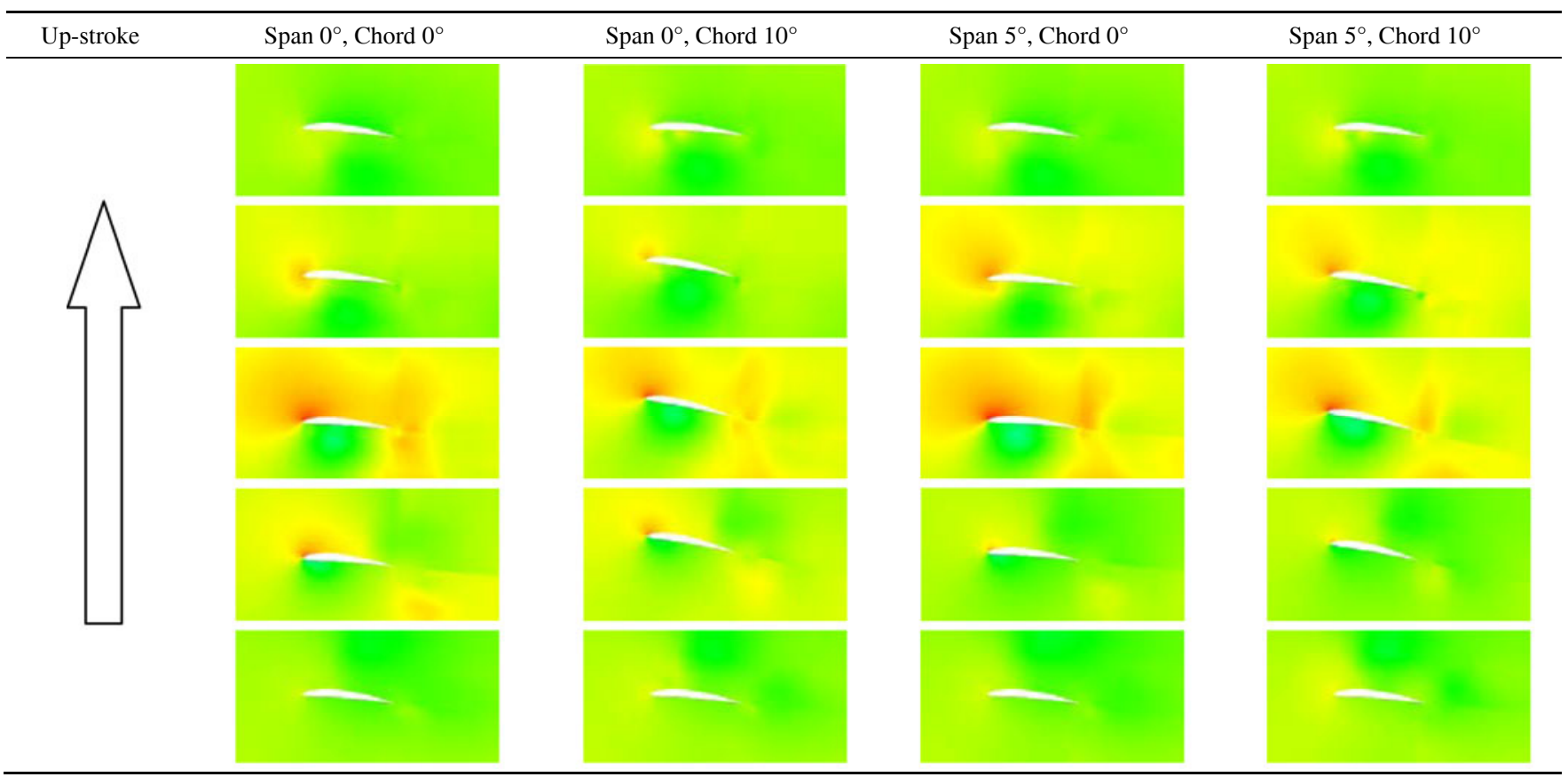


of Tables 1 and 2 are the same, which represent the top position of flapping cycle. Also the last rows of Tables 1 and 2 are the same, which represents the bottom position of flapping cycle. For the purpose of introduction, the deformation angle is set as span-wise $5^{\circ}$ and chord-wise $10^{\circ}$.

The effects of span-wise deformation could be approached in the comparison between columns 1 and 3 , or the comparison between columns 2 and 4 of Tables 1 and 2 . The span-wise deformation can enhance the leading edge pressure shown in the 4th row of Table 1 and 2 nd row of Table 2, which is not a good effect on lift or thrust. In the whole flapping cycle, the span-wise deformation does not show uniform effects.

The effects of chord-wise deformation could be approached in the comparison between columns 1 and 2, or the comparison between columns 3 and 4 of Tables 1 and 2 . The chordwise deformation can weaken the leading edge pressure and enhance the trailing edge pressure shown in the main part of flapping cycle except for the top and bottom positions, which is a good effect on thrust. Therefore, the chord-wise deformation is good to thrust.

The combining effects of both span-wise and chord-wise deformation could be approached in the comparison between columns 1 and 4 of Tables 1 and 2. The combining effect of column 4 is similar to the averaged effect of columns 2 and 3 .

\section{Conclusions}

The computational fluid dynamics method based on the preconditioned Navier-Stokes equations and chimera grid is used to study the effects of flapping-wing flexibility on the aerodynamic performance in span-wise and chord-wise directions. Four different kinds of deformation modes are investigated and analyzed. The results show that the large scale span-wise flexible deformation would depress aerodynamic performance of flapping-wing. However, small scale span-wise deformation can improve aerodynamic behavior slightly. Meanwhile, certain range chord-wise flexibility can enhance the aerodynamic performance of flapping- wing.

The combined deformation of span-wise and chord-wise can improve the aerodynamic performance of flapping-wing in bird-like scale, where the span-wise deformation should be controlled in a small range and the chord-wise defor- mation should be larger but with the limit that governed by different span-wise deformation.

The results suggest that the FMAV designers should design the flapping-wing with high stiffness leading edge and more flexible chord ribs. These structural characteristics can limit the span-wise deformation in a small range, and keep the chord-wise deformation in a suitable range, which makes structural deformation deflect to backward and cause stronger backward flow to increase thrust notably.

This work was supported by the Postdoctoral Science Foundation of China (20100481369).

1 Lin S Y, Hu J J. Aerodynamic performance study of flapping-wing flow fields. In: 23rd AIAA Applied Aerodynamics Conference, Toronto, Ontario Canada, 2005. AIAA 2005-4611

2 Zhang Y L, Zhao C X, Xu J L, et al. The effect of flapping-wing track to the aerodynamic performance (in Chinese). Chin Sci Bull (Chin Ver), 2006, 51: 634-640

3 Sun M, Tang J. Unsteady aerodynamic force generation by a model fruit fly wing in flapping motion. J Exp Biol, 2002, 205: 55-70

4 Xie H, Song W P, Song B F. Numerical solution of Navier-Stokes equations for flow over a flapping wing (in Chinese). J Northwest Polytech Univ, 2008, 26: 104-109

5 Yang W Q, Song B F, Song W P. Aerodynamic performance research of micro flapping-wing in low reynolds number flow (in Chinese). Acta Aerodyn Sin, 2011, 29: 32-38

6 Shao L M, Song B F, Xiong C, et al. Experimental investigation of flapping-wing MAV in wind tunnel (in Chinese). Acta Aeron Astron Sin, 2007, 28: 275-280

7 Jones K D, Platzer M F. An experimental and numerical investigation of flapping wing propulsion. AIAA 99-0995, 1999

8 Jones K D, Duggan S J, Platzer M F. Flapping-wing propulsion for a micro air vehicle. AIAA 2001-0126, 2001

9 Jones K D, Lund T C, Platzer M F. Experimental and computational investigation of flapping wing propulsion for micro air vehicles. Prog Aeron Astron, 2001, 195: 307-336

10 Wu P, Ifju P, Stanford B. Flapping wing structural deformation and thrust correlation study with flexible membrane wings. AIAA J, 2010, 48: 2111-2122

11 Chimakurth S K, Tang J, Palacios R, et al. Computational aeroelasticity framework for analyzing flapping wing micro air vehicles. AIAA J, 2009, 47: 1865-1878

12 Ho S, Nassef H, Pornsinsirirak N, et al. Unsteady aerodynamics and flow control for flapping wing flyers. Prog Aerospace Sci, 2003, 39: 635-681

13 Han Z H, Qiao Z D, Xiong J T. Development of an efficient viscous preconditioning method and its application to numerical simulation of flows over airfoils (in Chinese). J Northwest Polytech Univ, 2006, 24: 275-280

14 Fejek I, Nehera J. Experimental study of flapping wing lift and propulsion. Aeronaut J, 1980, 1: 28-33

Open Access This article is distributed under the terms of the Creative Commons Attribution License which permits any use, distribution, and reproduction in any medium, provided the original author(s) and source are credited. 